Scientific and Human Legacy of Julius Wess

International Journal of Modern Physics: Conference Series

Vol. 13 (2012) 118-126

(C) World Scientific Publishing Company

DOI: $10.1142 / \mathrm{S} 2010194512006782$

\title{
FUTS AND THE HIGGS-BOSON
}

\author{
SVEN HEINEMEYER \\ Instituto de Física de Cantabria (CSIC-UC), Edificio Juan Jorda, \\ Avda. de Los Castros s/n 39005 Santander, Spain \\ sven.heinemeyer@cern.ch \\ MYRIAM MONDRAGON \\ Inst. de Física, Universidad Nacional Autónoma de México, \\ Apdo. Postal 20-364, México 01000 D.F., México \\ myriam@fisica.unam.mx \\ GEORGE ZOUPANOS \\ Physics Department, National Technical University of Athens, \\ Zografou Campus: Heroon Polytechniou 9 \\ 15780 Zografou, Athens, Greece \\ george.zoupanos@cern.ch
}

Received 24 January 2012

Revised 6 March 2012

Keywords: Higgs mass, unified theories, supersymmetry

PACS numbers: $11.25 . \mathrm{Hf}, 123.1 \mathrm{~K}$

\section{Introduction}

The success of the Standard Model (SM) of Elementary Particle Physics is seriously limited by the presence of a plethora of free parameters. An even more disturbing fact is that the best bet for Physics beyond the SM namely the minimal supersymmetric extension of the SM (MSSM), which is expected to bring us one step further towards a more fundamental understanding of Nature, introduces around a hundred additional free parameters. To reduce the number of free parameters of a theory, and thus render it more predictive, one is usually led to introduce a symmetry. Grand Unified Theories (GUTs) are very good examples of such a procedure. ${ }^{1,2,3,4,5}$ For instance, in the case of minimal $S U(5)$, because of (approximate) gauge coupling unification, it was possible to reduce the gauge couplings by one and give a prediction for one of them. In fact, LEP data ${ }^{6}$ seem to suggest that a further symmetry, namely $N=1$ global supersymmetry (SUSY) ${ }^{7,8}$ should also be required to make the prediction viable. GUTs can also relate the Yukawa couplings among themselves, 
again $S U(5)$ provided an example of this by predicting the ratio $M_{\tau} / M_{b}{ }^{9}$ in the SM. Unfortunately, requiring more gauge symmetry does not seem to help, since additional complications are introduced due to new degrees of freedom, in the ways and channels of breaking the symmetry, and so on.

A natural extension of the GUT idea is to find a way to relate the gauge and Yukawa sectors of a theory, that is to achieve Gauge-Yukawa Unification (GYU). ${ }^{10,11,12}$ A symmetry which naturally relates the two sectors is supersymmetry, in particular $N=2$ SUSY. ${ }^{13}$ It turns out, however, that $N=2$ supersymmetric theories have serious phenomenological problems due to light mirror fermions. Also in superstring theories and in composite models there exist relations among the gauge and Yukawa couplings, but both kind of theories have phenomenological problems, which we are not going to address here.

Finite Unified Theories (FUTs) are $N=1$ supersymmetric Grand Unified Theories (GUTs) which can be made finite to all-loop orders, including the soft supersymmetry breaking sector. The constructed finite unified $N=1$ supersymmetric SU(5) GUTs predicted correctly from the dimensionless sector (Gauge-Yukawa unification), among others, the top quark mass. ${ }^{14,15}$ Eventually, the full theories can be made all-loop finite and their predictive power is extended to the Higgs sector and the s-spectrum. ${ }^{16}$ For a detailed discussion see Refs. 11, 17, 18. Here we limit ourselves to a brief review.

Consider a chiral, anomaly free, $N=1$ globally supersymmetric gauge theory based on a group $\mathrm{G}$ with gauge coupling constant $g$. The superpotential of the theory is given by

$$
W=\frac{1}{2} m^{i j} \Phi_{i} \Phi_{j}+\frac{1}{6} C^{i j k} \Phi_{i} \Phi_{j} \Phi_{k}
$$

where $m^{i j}$ (the mass terms) and $C^{i j k}$ (the Yukawa couplings) are gauge invariant tensors, and the matter field $\Phi_{i}$ transforms according to the irreducible representation $R_{i}$ of the gauge group $G$. All the one-loop $\beta$-functions of the theory vanish if the $\beta$-function of the gauge coupling $\beta_{g}^{(1)}$, and the anomalous dimensions of the Yukawa couplings $\gamma_{i}^{j(1)}$, vanish, i.e.

$$
\sum_{i} \ell\left(R_{i}\right)=3 C_{2}(G), \frac{1}{2} C_{i p q} C^{j p q}=2 \delta_{i}^{j} g^{2} C_{2}\left(R_{i}\right),
$$

where $\ell\left(R_{i}\right)$ is the Dynkin index of $R_{i}$, and $C_{2}(G)$ is the quadratic Casimir invariant of the adjoint representation of $G$. A theorem given in Ref. 19, 20, 21, 22 then guarantees the vanishing of the $\beta$-functions to all-orders in perturbation theory. This requires that, in addition to the one-loop finiteness conditions (2), the Yukawa couplings are reduced in favour of the gauge coupling.

In the soft breaking sector, the one- and two-loop finiteness for the trilinear terms $h^{i j k}$ can be achieved by ${ }^{23}$

$$
h^{i j k}=-M C^{i j k}+\cdots=-M \rho_{(0)}^{i j k} g+O\left(g^{5}\right) .
$$


It was also found that the soft supersymmetry breaking (SSB) scalar masses in Gauge-Yukawa and finite unified models satisfy a sum rule 24,25

$$
\frac{\left(m_{i}^{2}+m_{j}^{2}+m_{k}^{2}\right)}{M M^{\dagger}}=1+\frac{g^{2}}{16 \pi^{2}} \Delta^{(2)}+O\left(g^{4}\right)
$$

for $\mathrm{i}, \mathrm{j}, \mathrm{k}$, where $\Delta^{(2)}$ is the two-loop correction, which vanishes when all the soft scalar masses are the same at the unification point.

\section{2. $S U(5)$ Finite Unified Theories}

We will examine here all-loop Finite Unified theories with $S U(5)$ gauge group, where the reduction of couplings has been applied to the third generation of quarks and leptons. An extension to three families, and the generation of quark mixing angles and masses in Finite Unified Theories has been addressed in Ref. 26, where several examples are given. These extensions are not considered here. The particle content of the models we will study consists of the following supermultiplets: three $(\overline{\mathbf{5}}+\mathbf{1 0})$, needed for each of the three generations of quarks and leptons, four $(\overline{\mathbf{5}}+\mathbf{5})$ and one 24 considered as Higgs supermultiplets. When the gauge group of the finite GUT is broken the theory is no longer finite, and we will assume that we are left with the MSSM.

A predictive Gauge-Yukawa unified $S U(5)$ model which is finite to all orders, should have the following properties:

(1) One-loop anomalous dimensions are diagonal, i.e., $\gamma_{i}^{(1) j} \propto \delta_{i}^{j}$.

(2) Three fermion generations, in the irreducible representations $\overline{\mathbf{5}}_{i}, \mathbf{1 0}_{i}(i=1,2,3)$, which obviously should not couple to the adjoint 24 .

(3) The two Higgs doublets of the MSSM should mostly be made out of a pair of Higgs quintet and anti-quintet, which couple to the third generation.

In the following we discuss two versions of the all-order finite model. The model of Ref. 14, 15, which will be labeled $\mathbf{A}$, and a slight variation of this model (labeled B), which can also be obtained from the class of the models suggested in Ref. 27 with a modification to suppress non-diagonal anomalous dimensions.

The superpotential which describes the two models takes the form ${ }^{14,15,28}$

$$
\begin{aligned}
W & =\sum_{i=1}^{3}\left[\frac{1}{2} g_{i}^{u} \mathbf{1 0}_{i} \mathbf{1 0}_{i} H_{i}+g_{i}^{d} \mathbf{1 0}_{i} \overline{\mathbf{5}}_{i} \bar{H}_{i}\right] \\
& +g_{23}^{u} \mathbf{1 0}_{2} \mathbf{1 0}_{3} H_{4}+g_{23}^{d} \mathbf{1 0}_{2} \overline{\mathbf{5}}_{3} \bar{H}_{4}+g_{32}^{d} \mathbf{1 0}_{3} \overline{\mathbf{5}}_{2} \bar{H}_{4} \\
& +\sum_{a=1}^{4} g_{a}^{f} H_{a} \mathbf{2 4} \bar{H}_{a}+\frac{g^{\lambda}}{3}(\mathbf{2 4})^{3}
\end{aligned}
$$

where $H_{a}$ and $\bar{H}_{a} \quad(a=1, \ldots, 4)$ stand for the Higgs quintets and anti-quintets.

The main difference between model $\mathbf{A}$ and model $\mathbf{B}$ is that two pairs of Higgs quintets and anti-quintets couple to the $\mathbf{2 4}$ in $\mathbf{B}$, so that it is not necessary to mix them with $H_{4}$ and $\bar{H}_{4}$ in order to achieve the triplet-doublet splitting after the 
symmetry breaking of $S U(5) .{ }^{28}$ Thus, although the particle content is the same, the solutions to the finiteness equations and the sum rules are different, which will reflect in the phenomenology.

The non-degenerate and isolated solutions to $\gamma_{i}^{(1)}=0$ for model FUTA, which are the boundary conditions for the Yukawa couplings at the GUT scale, are:

$$
\begin{aligned}
& \left(g_{1}^{u}\right)^{2}=\frac{8}{5} g^{2},\left(g_{1}^{d}\right)^{2}=\frac{6}{5} g^{2},\left(g_{2}^{u}\right)^{2}=\left(g_{3}^{u}\right)^{2}=\frac{8}{5} g^{2}, \\
& \left(g_{2}^{d}\right)^{2}=\left(g_{3}^{d}\right)^{2}=\frac{6}{5} g^{2},\left(g_{23}^{u}\right)^{2}=0,\left(g_{23}^{d}\right)^{2}=\left(g_{32}^{d}\right)^{2}=0, \\
& \left(g^{\lambda}\right)^{2}=\frac{15}{7} g^{2},\left(g_{2}^{f}\right)^{2}=\left(g_{3}^{f}\right)^{2}=0,\left(g_{1}^{f}\right)^{2}=0,\left(g_{4}^{f}\right)^{2}=g^{2} .
\end{aligned}
$$

In the dimensionful sector, the sum rule gives us the following boundary conditions at the GUT scale for this model: ${ }^{28,29,30}$

$$
m_{H_{u}}^{2}+2 m_{\mathbf{1 0}}^{2}=m_{H_{d}}^{2}+m_{\frac{5}{5}}^{2}+m_{\mathbf{1 0}}^{2}=M^{2}
$$

and thus we are left with only three free parameters, namely $m_{\overline{\mathbf{5}}} \equiv m_{\overline{\mathbf{5}}_{3}}, m_{\mathbf{1 0}} \equiv m_{\mathbf{1 0}_{3}}$ and $M$.

For the model FUTB the non-degenerate and isolated solutions to $\gamma_{i}^{(1)}=0$ give us:

$$
\begin{aligned}
& \left(g_{1}^{u}\right)^{2}=\frac{8}{5} g^{2},\left(g_{1}^{d}\right)^{2}=\frac{6}{5} g^{2},\left(g_{2}^{u}\right)^{2}=\left(g_{3}^{u}\right)^{2}=\frac{4}{5} g^{2}, \\
& \left(g_{2}^{d}\right)^{2}=\left(g_{3}^{d}\right)^{2}=\frac{3}{5} g^{2},\left(g_{23}^{u}\right)^{2}=\frac{4}{5} g^{2},\left(g_{23}^{d}\right)^{2}=\left(g_{32}^{d}\right)^{2}=\frac{3}{5} g^{2}, \\
& \left(g^{\lambda}\right)^{2}=\frac{15}{7} g^{2},\left(g_{2}^{f}\right)^{2}=\left(g_{3}^{f}\right)^{2}=\frac{1}{2} g^{2},\left(g_{1}^{f}\right)^{2}=0,\left(g_{4}^{f}\right)^{2}=0,
\end{aligned}
$$

and from the sum rule we obtain:

$$
m_{H_{u}}^{2}+2 m_{\mathbf{1 0}}^{2}=M^{2}, m_{H_{d}}^{2}-2 m_{\mathbf{1 0}}^{2}=-\frac{M^{2}}{3}, m_{\overline{\mathbf{5}}}^{2}+3 m_{\mathbf{1 0}}^{2}=\frac{4 M^{2}}{3},
$$

i.e., in this case we have only two free parameters $m_{\mathbf{1 0}} \equiv m_{\mathbf{1 0}_{3}}$ and $M$.

\section{Predictions of the $S U(5)$ Models}

We confront now the predictions of the four models with the experimental data, starting with the heavy quark masses (see Refs. 16, 18 for more details). Since the gauge symmetry is spontaneously broken below $M_{\mathrm{GUT}}$, the finiteness conditions do not restrict the renormalization properties at low energies, and all it remains are boundary conditions on the gauge and Yukawa couplings (6), the $h=-M C$ relation eq.(3), and the soft scalar-mass sum rule (4) at $M_{\mathrm{GUT}}$, as applied in each of the models. Thus we examine the evolution of these parameters according to their RGEs up to two-loops for dimensionless parameters and at one-loop for dimensionful ones with the relevant boundary conditions. Below $M_{\mathrm{GUT}}$ their evolution is assumed to be governed by the MSSM. We further assume a unique supersymmetry breaking scale $M_{s}$ and therefore below that scale the effective theory is just the SM. 
As a first step, we compare the predictions of the two models, FUTA and FUTB (both with $\mu>0$ and $\mu<0$ ), with the experimental values of the top and bottom quark masses. We use for the top quark the value for the pole mass ${ }^{31}$

$$
M_{t}^{\text {exp }}=(173.1 \pm 1.3)
$$

where we notice that the theoretical values for $M_{t}$ may suffer from a correction of $\sim 4 \%{ }^{11}$ For the bottom quark mass we use the value at $M_{Z}{ }^{32}$

$$
m_{b}\left(M_{Z}\right)=2.83 \pm 0.10 G e V
$$

to avoid errors that come from the furhter running from the $M_{Z}$ to the $m_{b}$ mass, and where we have taken the $\Delta b$ effects into account.

From fig. 1 it is clear that the model FUTB with $\mu<0$ is the only one where both top and bottom quark masses lie within experimental limits. In this case the value of $\tan \beta$ is found to be $\sim 48$. Thus, we will concentrate now on the results
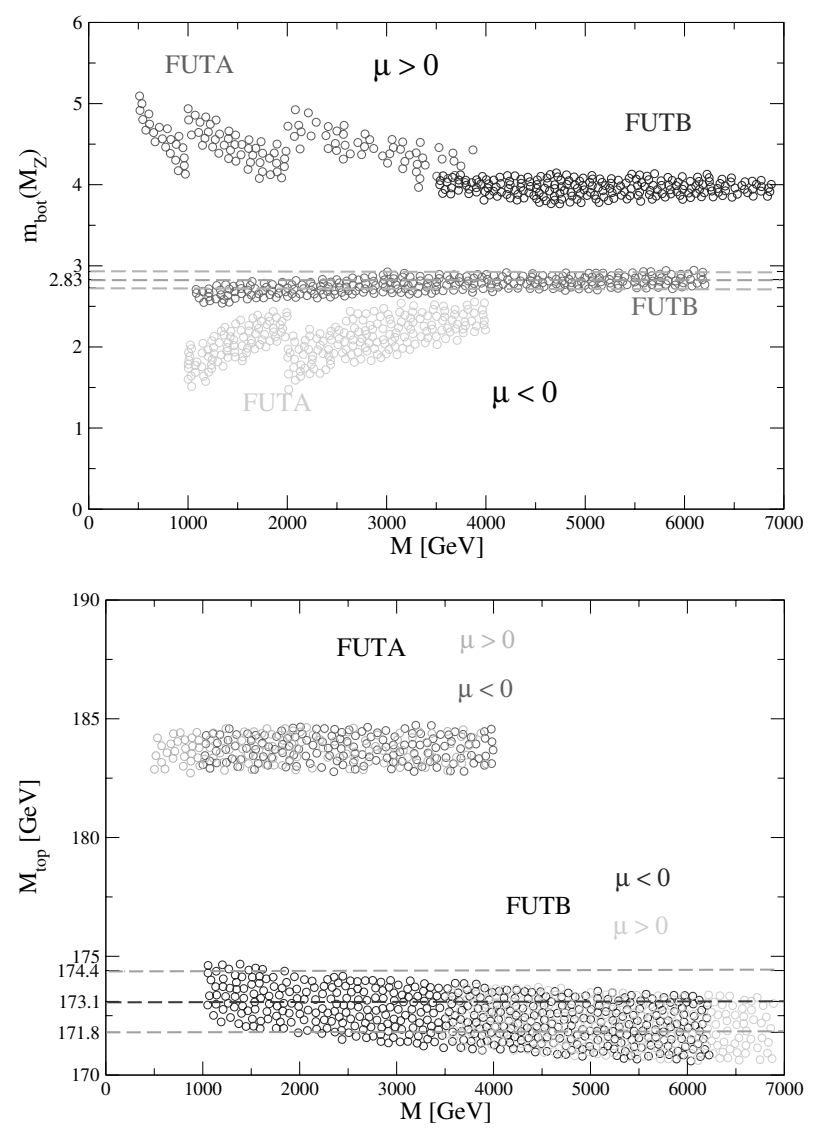

Fig. 1. $m_{b}\left(M_{Z}\right)$ as function of $M$ (top) and $M_{t o p}$ as function of $M$ (bottom) for models FUTA and FUTB, for $\mu<0$ and $\mu>0$. 
for FUTB, $\mu<0$. In the case where all the soft scalar masses are universal at the unfication scale, there is no region of $M$ below $\mathcal{O}$ (few $\mathrm{TeV})$ in which $m_{\tilde{\tau}}>m_{\chi^{0}}$ is satisfied (where $m_{\tilde{\tau}}$ is the lightest $\tilde{\tau}$ mass, and $m_{\chi^{0}}$ the lightest neutralino mass). This problem can be solved naturally, thanks to the sum rule (9).

Furthermore, we impose the conditions of successful radiative electroweak symmetry breaking, $m_{\tilde{\tau}}^{2}>0$ and $m_{\tilde{\tau}}>m_{\chi^{0}}$, plus the constraints coming from B physics, namely the experimental bounds on $\mathrm{BR}(\mathrm{b} \rightarrow \mathrm{s} \gamma)$ and $\mathrm{BR}\left(\mathrm{B}_{\mathrm{s}} \rightarrow \mu^{+} \mu^{-}\right)$(which we have evaluated with Micromegas $\left.{ }^{33}\right)$. This way, we find a prediction for the lightest Higgs mass and the s-spectra. From the analysis we find that the lightest observable particle (LOSP) is either the stau or the second lightest neutralino, with mass starting around $\sim 500 \mathrm{GeV}$.

The prediction of the lightest Higgs boson mass as a function of $M$ is shown in Fig. 2. The light points shown are in agreement with the two $B$-physics observables listed above. The lightest Higgs mass ranges in

$$
M_{h} \sim 121-126 \mathrm{GeV},
$$

where the uncertainty comes from variations of the soft scalar masses, and from finite (i.e. not logarithmically divergent) corrections in changing renormalization scheme. To this value one has to add $\pm 3 \mathrm{GeV}$ coming from unknown higher order corrections. ${ }^{34}$ We have also included a small variation, due to threshold corrections at the GUT scale, of up to $5 \%$ of the FUT boundary conditions. Thus, taking into account the $B$ physics constraints results naturally in a light Higgs boson that fulfills the LEP bounds. ${ }^{35,36}$ See Refs. 18,16 for a more detailed analysis.

The prediction for the lightest Higgs mass, $M_{h}$ as function of $M$ for the model FUTB with $\mu<0$ is shown in Fig. 2. The full particle spectrum of model FUTB with $\mu<0$, again compliant with quark mass constraints and the $B$ physics observables is shown in Fig. 3. The masses of the particles increase with increasing values of the unified gaugino mass $M$.

One can see that large parts of the spectrum are in the kinematic reach of the LHC. A numerical example of such a light spectrum is shown in Table 1. The colored

Table 1. A representative spectrum of a light FUTB, $\mu<0$ spectrum.

\begin{tabular}{cc|cc}
\hline Mbot $\left(M_{Z}\right)$ & $2.71 \mathrm{GeV}$ & Mtop & $172.2 \mathrm{GeV}$ \\
Mh & $123.1 \mathrm{GeV}$ & MA & $680 \mathrm{GeV}$ \\
MH & $679 \mathrm{GeV}$ & MH$^{ \pm}$ & $685 \mathrm{GeV}$ \\
Stop1 & $1876 \mathrm{GeV}$ & Stop2 & $2146 \mathrm{GeV}$ \\
Sbot1 & $1849 \mathrm{GeV}$ & Sbot2 & $2117 \mathrm{GeV}$ \\
Mstau1 & $635 \mathrm{GeV}$ & Mstau2 & $867 \mathrm{GeV}$ \\
Char1 & $1072 \mathrm{GeV}$ & Char2 & $1597 \mathrm{GeV}$ \\
Neu1 & $579 \mathrm{GeV}$ & Neu2 & $1072 \mathrm{GeV}$ \\
Neu3 & $1591 \mathrm{GeV}$ & Neu4 & $1596 \mathrm{GeV}$ \\
M1 & $580 \mathrm{GeV}$ & M2 & $1077 \mathrm{GeV}$ \\
Mgluino & $2754 \mathrm{GeV}$ & & \\
\hline
\end{tabular}




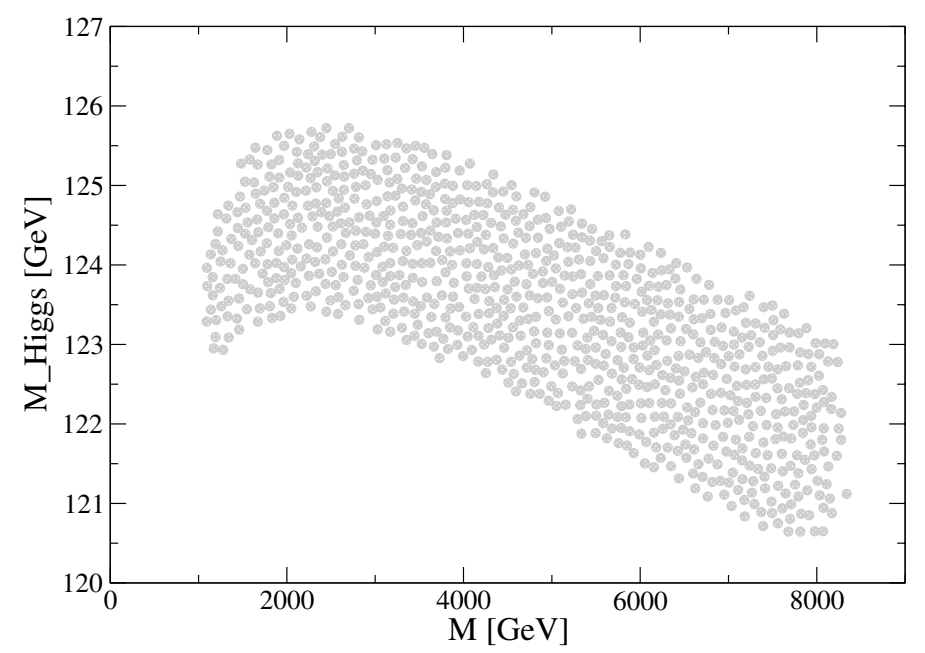

Fig. 2. The lighest Higgs mass $M_{h}$ as function of the unified gaugino mass $M$.

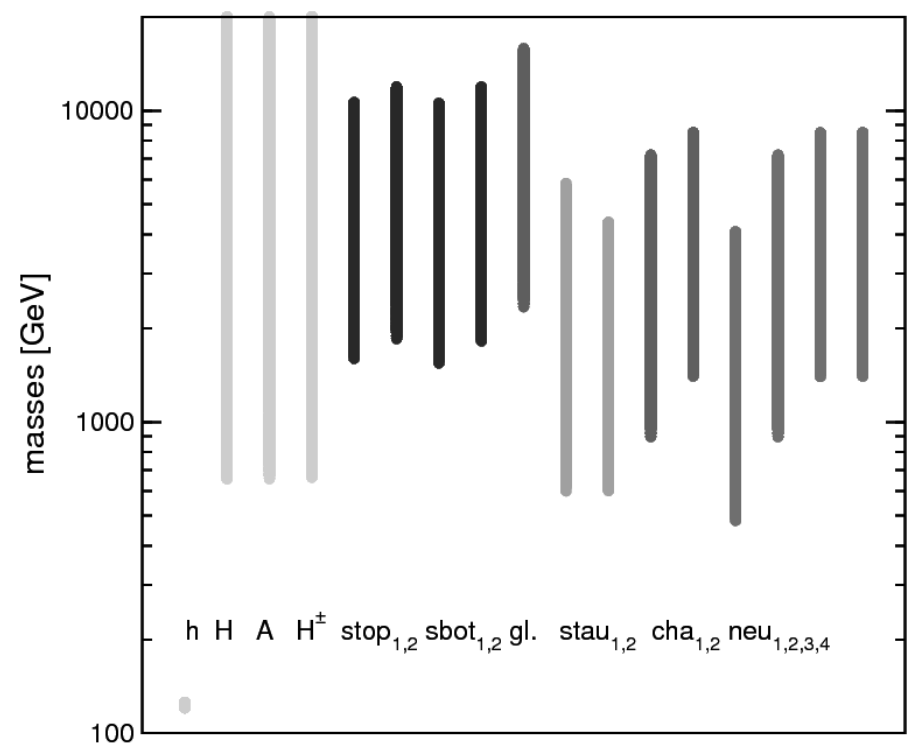

Fig. 3. The particle spectrum of model FUTB with $\mu<0$, where the points shown are in agreement with the quark mass constraints and the $B$-physics observables. The light points on the left are the various Higgs boson masses. The dark points following are the two scalar top and bottom masses, followed by the gluino and the lighter scalar tau masses. The darker points to the right are the two chargino masses followed by the lighter shaded points indicating the neutralino masses. 
part of this spectrum as well as the lightest Higgs boson should be (relatively easily) accessible at the LHC.

\section{Acknowledgments}

Dedicated to the memory of our great friend and teacher in all respects Julius Wess.

This work is partially supported by the NTUA's programme supporting basic research PEBE 2009 and 2010, and the European Union's ITN programme "UNILHC" PITN-GA-2009-237920. Supported also by mexican PAPIIT grant IN113712.

\section{References}

1. J. C. Pati and A. Salam,Phys. Rev. Lett. 31, 661 (1973).

2. H. Georgi and S. L. Glashow, Phys. Rev. Lett. 32, 438 (1974).

3. H. Georgi, H. R. Quinn and S. Weinberg, Phys. Rev. Lett. 33, 451 (1974).

4. H. Fritzsch and P. Minkowski, Ann. Phys. 93, 193 (1975).

5. H. Georgi, Particles and Fields: Williamsburg 1974, AIP Conference Proceedings No. 23, ed. C. E. Carlson, (American Institute of Physics, New York, 1974).

6. U. Amaldi, W. de Boer and H. Furstenau, Phys. Lett. B 260, 447 (1991).

7. S. Dimopoulos and H. Georgi, Nucl. Phys. B 193, 150 (1981).

8. N. Sakai, Zeit. Phys. C 11, 153 (1981).

9. A. J. Buras, J. R. Ellis, M. K. Gaillard and D. V. Nanopoulos, Nucl. Phys. B 135, 66 (1978).

10. J. Kubo, M. Mondragon, M. Olechowski and G. Zoupanos, Nucl. Phys. B 479, 25 (1996).

11. J. Kubo, M. Mondragon and G. Zoupanos, Acta Phys. Polon. B 27, 3911 (1997).

12. T. Kobayashi, J. Kubo, M. Mondragon and G. Zoupanos, Acta Phys. Polon. B 30, 2013 (1999).

13. P. Fayet, Nucl. Phys. B 149, 137 (1979).

14. D. Kapetanakis, M. Mondragon and G. Zoupanos, Z. Phys. C 60, 181 (1993).

15. M. Mondragon and G. Zoupanos, Nucl. Phys. Proc. Suppl. 37C, 98 (1995).

16. S. Heinemeyer, M. Mondragon and G. Zoupanos, JHEP 07, 135 (2008).

17. T. Kobayashi, J. Kubo, M. Mondragon and G. Zoupanos, Surveys High Energ. Phys. 16, 87 (2001).

18. S. Heinemeyer, M. Mondragon and G. Zoupanos, SIGMA 6, 049 (2010).

19. C. Lucchesi, O. Piguet and K. Sibold, Helv. Phys. Acta 61, 321 (1988).

20. O. Piguet and K. Sibold, Int. J. Mod. Phys. A 1, 913 (1986).

21. O. Piguet and K. Sibold, Phys. Lett. B 177, 373 (1986).

22. C. Lucchesi and G. Zoupanos, Fortschr. Phys. 45, 129 (1997).

23. I. Jack and D. R. T. Jones, Phys. Lett. B 333, 372 (1994).

24. T. Kobayashi, J. Kubo and G. Zoupanos, Phys. Lett. B 427, 291 (1998).

25. J. Kubo, M. Mondragon and G. Zoupanos, Nucl. Phys. B 424, 291 (1994).

26. K. S. Babu, T. Enkhbat and I. Gogoladze, Phys. Lett. B 555, 238 (2003).

27. D. I. Kazakov, M. Y. Kalmykov, I. N. Kondrashuk and A. V. Gladyshev, Nucl. Phys. B 471, 389 (1996).

28. T. Kobayashi, J. Kubo, M. Mondragon and G. Zoupanos, Nucl. Phys. B 511, 45 (1998). 
29. D. R. T. Jones and S. Raby, Phys. Lett. B 143, 137 (1984).

30. J. Leon, J. Perez-Mercader, M. Quiros and J. Ramirez-Mittelbrunn, Phys. Lett. B 156, 66 (1985).

31. (2009), Tevatron Electroweak Working Group, arXiv:0903.2503 [hep-ex].

32. C. Amsler et al., Phys. Lett. B 667, 1 (2008).

33. G. Belanger, F. Boudjema, A. Pukhov and A. Semenov, Comput. Phys. Commun. 149, 103 (2002).

34. G. Degrassi, S. Heinemeyer, W. Hollik, P. Slavich and G. Weiglein, Eur. Phys. J. C 28, 133 (2003).

35. R. Barate et al., Phys. Lett. B 565, 61 (2003).

36. S. Schael et al., Eur. Phys. J. C 47, 547 (2006). 